\title{
Propostas de material didático para o fomento da autonomia na aprendizagem de língua inglesa
}

\author{
Walkyria Magno e Silva \\ Universidade Federal do Pará \\ Edwiges C. de Souza Fernandes \\ Universidade do Estado do Pará \\ Marcus de Souza Araújo \\ Universidade Federal do Pará
}

\section{Resumo}

Nos últimos anos, a autonomia na aprendizagem de línguas estrangeiras (LE) passou a ter um lugar de destaque no contexto educacional brasileiro. Neste artigo, pretendemos propor materiais didáticos, tarefas e estratégias de aprendizagem de língua inglesa que toquem os interesses de aprendentes ${ }^{1}$ diversos, catalisando a busca autônoma por conhecimentos. Acreditarmos, assim, que todos esses recursos são capazes de promover práticas de escolhas envolvendo tomada de consciência, posicionamento crítico e reflexão, as quais fornecem ao aprendente oportunidades para trabalhar de forma mais independente fora do contexto de sala de aula.

Palavras chave: Autonomia; aprendizagem de línguas estrangeiras; materiais didáticos.

\begin{abstract}
In recent years, in the Brazilian educational context, a special emphasis has been given to autonomy in foreign language learning. This paper offers some options for using pedagogical materials, tasks and strategies in ways that arouse English language learners' interests, thus enhancing their desire to use their own autonomy to search for more knowledge. In this paper, we also argue that all these resources serve to promote practices of choices involving awareness, positioning and critical reflection which provide learners with opportunities to work more independently outside the context of the classroom.
\end{abstract}

Keywords: Autonomy; foreign language learning; pedagogical materials.

\footnotetext{
${ }^{1}$ Neste texto utilizamos o termo aprendente no lugar de aprendiz por sua conotação mais ativa e participativa. Este termo tem sido empregado em diversas publicações recentes na área da Linguística Aplicada no Brasil.
} 


\section{INTRODUÇÃO}

Autonomia passou a ser amplamente discutida no contexto educacional a partir do final dos anos 60, através de movimentos educacionais na Europa e América do Norte, e por muitos anos foi associada à educação de adultos. Nos últimos anos, porém, a autonomia na aprendizagem de línguas estrangeiras (LE) passou a ter um lugar de destaque no contexto educacional brasileiro. Autonomia é aqui entendida como uma capacidade que pode ser desenvolvida (Benson, 2001) e como um sistema complexo (Paiva, 2005). Os estudos desenvolvidos na área enfocaram inicialmente o papel do aluno e depois o papel do professor, sendo que poucos trabalhos se dedicam a analisar os materiais didáticos (MDs) sob essa ótica.

Sabe-se que a aprendizagem é intensificada quando as necessidades dos aprendentes são levadas em consideração na elaboração do currículo e na aplicação da metodologia, pois aprendentes autônomos são capazes de exercer liberdade de escolha em relação às fontes e atividades de aprendizagem e assim influir positivamente tanto na qualidade quanto na quantidade dessa aprendizagem (Benson, 2001). É certo que, em havendo um professor autonomizador, quaisquer MDs podem ser transformados e adaptados de forma a levarem o aprendente a buscar conhecimento por si próprio. No entanto, é também provável que em grande parte os MDs atuem como "camisas de força” no planejamento pedagógico. Desta forma, é necessário promover o envolvimento do aprendente com o MD, pressupondo-se que, se houver esse interesse, ele não se contentará apenas com o que lhe foi proposto em sala de aula e partirá do material apresentado para a expansão dos conteúdos transformando os objetos de aprendizagem em materiais de uso na vida real. Buscamos esse envolvimento por meio da inclusão do aprendente no processo de tomada de decisões sobre sua aprendizagem, ou seja, as escolhas que ele deve fazer em relação às atividades que vai executar.

Neste artigo apresentamos materiais que a) sejam passíveis de tocar pontos de interesse geral dos aprendentes; b) facilitem a ação do professor em seu contexto de ensino; e c) levem o aprendente a complementar seus conhecimentos com materiais que ele busque autonomamente a partir da aula lecionada. Os MDs aqui apresentados não se constituem em 
fórmulas nem mesmo 'receitas' prontas, mas tarefas ${ }^{2}$ significativas que levem o aprendente a gerenciar sua própria aprendizagem dentro do contexto social em que atua, como forma de ajudá-lo a encontrar soluções para suas dificuldades na língua-alvo. Além disso, o uso desses MDs pode incentivá-lo a adquirir confiança em sua própria capacidade de aprender. Os itens que serão demonstrados inscrevem-se na tendência de os MDs serem cada vez mais moldáveis a diferentes situações de aprendizagem, atendo-se aos pontos principais, liberando, assim, tempo para o uso de materiais adicionais, estes últimos altamente fomentadores de autonomia.

Organizamos nosso artigo a partir da apresentação do termo autonomia na aprendizagem em LE. Em seguida, abordamos sobre a produção de material didático. Por fim, apresentamos algumas propostas de material didático para o fomento da aprendizagem autônoma.

\section{AUTONOMIA NA APRENDIZAGEM DE LÍNGUAS: DEFINIÇÕES}

A década de 70 foi o marco para o desenvolvimento da autonomia na Europa onde Henry Holec (1981) e um grupo de pesquisadores no campo de ensino e aprendizagem de línguas desenvolveram importantes pesquisas no Centre de Recherches et d'Application en Langues (CRAPEL) ${ }^{3}$. Desde então, os estudos referentes ao fomento da autonomia passaram a ser objeto de estudo de vários pesquisadores no mundo, inclusive no Brasil.

$\mathrm{Na}$ literatura especializada encontramos diversas definições específicas para autonomia. Em Dickinson (1987) encontramos os seguintes termos que estão relacionados ao aprendizado autônomo: auto-instrução, autodireção, semiautonomia, materiais de autoacesso, aprendizagem de autoacesso e instrução individualizada. Apesar da existência das diferentes

\footnotetext{
2 Entendemos tarefa conforme o proposto por Breen (1987:23): “qualquer esforço estrutural para a aprendizagem de língua que tenha: um objetivo específico, conteúdo apropriado, um procedimento específico de trabalho e uma variedade de resultados para aqueles que executam a tarefa.(...) Tarefas referem-se a vários planos de trabalho que tenham o propósito abrangente de facilitar a aprendizagem de línguas e podem variar desde tipos de exercícios simples e breves ou atividades mais longas e mais complexas, como simulações para a solução de problemas ou tomadas de decisão”.

${ }^{3}$ Este centro tem como objetivo incentivar a aprendizagem autônoma autodirigida. Ali os alunos encontram os mais diversos materiais impressos, materiais de áudio e vídeo, todos na língua alvo, assim como as mais variadas oportunidades de treinamento autônomo estimulado pela ajuda de monitores. Pelas valiosas contribuições, o CRAPEL ainda continua a ser um grande centro de pesquisa em autonomia nos dias atuais.
} 
definições sobre o assunto, consideramos que a promoção da autonomia está relacionada à conscientização de habilidades, atitudes e estratégias de aprendizagem necessárias para o aprendente lidar com situações nas quais ele deve tomar decisões relevantes para sua própria aprendizagem, tornando-se, deste modo, um agente na busca e construção de conhecimento. Sendo assim, concordando com o pensamento de Benson (2001), consideramos que autonomia é a capacidade de alguém controlar sua própria aprendizagem, pois não basta apenas o aprendente decidir o local, o conteúdo e seu ritmo de estudo, mas é preciso que ele esteja diretamente envolvido na definição de objetivos e na avaliação do processo, por exemplo. Benson e Huang (2008), na mesma perspectiva, mencionam que autonomia é uma capacidade subjacente do indivíduo, que pode ser desenvolvida ou suprimida ou até mesmo distorcida pela educação institucional.

Dickinson (1994) afirma que autonomia não é (a) uma licença para comportar-se sem restrições; (b) uma questão de cenário físico de aprendizagem nem (c) uma ameaça ao trabalho do professor. Nesse último item, ressaltamos que o papel do professor é fundamental para o fomento da autonomia do aprendente na aprendizagem de línguas, pois professores bem preparados encorajarão seus alunos a trabalhar independentemente na sala de aula e fora dela. Desta forma, o professor passa a ser um facilitador da aprendizagem.

Magno e Silva (2008) também se refere à promoção da autonomia como uma capacidade existente no indivíduo e que precisa ser desenvolvida. Por isso, a conscientização de estratégias é fator fundamental para o fomento da autonomia; no entanto, não basta apenas ensiná-las, o aprendente deve estar consciente dos fatores que afetam sua aprendizagem para decidir sobre a melhor estratégia ou a mais apropriada a ser usada em um determinado contexto. Desta forma, ele se responsabiliza por sua própria aprendizagem, gerenciando seu tempo e seu ritmo e respeitando, assim, suas preferências e necessidades individuais.

Entre as múltiplas definições apresentadas na literatura, citadas anteriormente, preferimos destacar neste estudo a posição de Benson (2001) e Magno e Silva (2008) de que autonomia é "uma capacidade multidimensional que assumirá formas diferentes para indivíduos diferentes, e até mesmo para o mesmo indivíduo em épocas diferentes” (Benson, 2001:47). Essa capacidade envolve tomada de consciência, posicionamento crítico e reflexão no ensino e aprendizagem de línguas estrangeiras, tanto por parte do professor quanto do aprendente. 


\section{PRODUÇÃO DE MATERIAIS DIDÁTICOS PARA O FOMENTO DE AUTONOMIA:}

\section{Eixos/critérios de avaliação do potencial de autonomização em MDS}

A partir do exposto a respeito de autonomia na seção anterior, faremos uma tentativa de estabelecer critérios por meio dos quais os professores possam ser ajudados a perceber o potencial de autonomização, presente nos materiais didáticos que utilizam. Alternativamente, esses critérios podem ser utilizados também na própria seleção dos MDs, desde que o professor esteja engajado nesse propósito. Apresentamos, a seguir, uma proposta que melhor define esses critérios.

\begin{tabular}{|l|l|l|}
\hline \multicolumn{1}{|c|}{ Critério } & \multicolumn{1}{|c|}{ Ação } & \multicolumn{1}{c|}{ Professor } \\
\hline \multirow{2}{*}{ Escolha } & O que fazer & Dá ao aluno opções de tarefas \\
\cline { 2 - 3 } Expansão & $\begin{array}{l}\text { Como fazer } \\
\text { conhecimentos }\end{array}$ & $\begin{array}{l}\text { Dá ao aluno opções de como } \\
\text { realizar uma mesma tarefa }\end{array}$ \\
\hline Transformação & $\begin{array}{l}\text { Fazer algo diferente a partir } \\
\text { do MD }\end{array}$ & $\begin{array}{l}\text { Indica possibilidades de } \\
\text { acréscimo de informações a } \\
\text { serem buscadas pelos alunos }\end{array}$ \\
$\begin{array}{l}\text { Muda o propósito, o conteúdo ou } \\
\text { a forma proposta pelo MD }\end{array}$ \\
\hline
\end{tabular}

\section{Quadro 1: Eixos de produção de material didático}

Ao proporcionar opções de escolha, seja do que fazer ou de como executar uma tarefa, o professor estará dividindo com os aprendentes a responsabilidade pela aprendizagem e, assim, fazendo com que seja criado um vínculo entre eles e suas tarefas.

Ao indicar a possibilidade de, a partir de um conhecimento adquirido, os aprendentes buscarem mais informações, o professor estará contribuindo para a autonomização deles, através de práticas que os levarão muito além da sala de aula.

Mesmo tarefas corriqueiras constantes no MD utilizado em instituições diversas podem ser transformadas pelo professor em tarefas mais interessantes para o público alvo. Essa transformação terá como princípio norteador o perfil dos estudantes e seus interesses. 
Ao realizar tarefas dentro de seu campo de interesses, os aprendentes tomá-las-ão como exemplos de outras que poderão fazer sozinhos a partir do MD de seu curso.

Com base nesses critérios apresentamos a seguir exemplos de tarefas fomentadoras da autonomia dos alunos.

\section{Propostas de material didático para o fomento de autonomia}

Nesta seção, daremos exemplos de tarefas que podem ser realizadas em um primeiro momento com a orientação dos professores para fomento da aprendizagem autônoma. Em seguida, os alunos poderão repeti-las sozinhos, sem supervisão. As tarefas propostas não são 'receitas' pré-fabricadas, mas tarefas nas quais o aprendente poderá gerenciar sua própria aprendizagem fora de sala de aula, levando em consideração suas necessidades para aprimorar a língua-alvo.

\section{TAREFA 1: Avaliar web sites}

Esta tarefa está dividida em dois momentos, os quais serão brevemente descritos a seguir.

\section{Avaliar web sites (parte 1)}

A tarefa tem como objetivo levar o aluno a avaliar web sites de maneira consciente. Para a realização da tarefa, o professor solicita ao aluno que ele escolha um texto em um site relacionado a qualquer assunto de sua preferência. Caso o professor trabalhe com alunos de cursos de Línguas para Fins Específicos, poderá haver a alternativa de se consultar sites relacionados diretamente à área de estudo do aluno. Para isso, o aluno estará de posse de um checklist (ver anexo ).

A avaliação dos sites poderá ser realizada individualmente ou em grupo, dependendo do número de alunos de cada turma. Em seguida, o professor solicita que os alunos compartilhem suas impressões ao avaliar os sites escolhidos.

\section{Avaliar web sites (parte 2)}

Na segunda parte da tarefa, o professor apresenta cinco opções de avaliação de sites em que o aluno poderá navegar para se familiarizar com a proposta de cada autor, porém ele 
deverá escolher apenas um dos sites para a realização da tarefa. Nesta segunda parte, o aluno escolhe outro site sobre qualquer assunto. Os sites sugeridos são apresentados a seguir:

Evaluating web sites:

(a) ClassZone - McDougal Littell (editora)

http://www.classzone.com/books/research_guide/page_build.cfm?content=web_eval\&sta $\underline{\text { te}}=$ none

(b) University of California Berkeley Library:

http://www.lib.berkeley.edu/TeachingLib/Guides/Internet/Evaluate.html

(c) Teaching with technology (Livro on-line de Peter Desberg e Farah Fisher):

http://www.abacon.com/twt/cred.html

(d) Discovery Education:

http://school.discoveryeducation.com/schrockguide/eval.html

(e) Johns Hopkins University - The Sheridan Libraries:

http://www.library.jhu.edu/researchhelp/general/evaluating/

A ideia é direcionar o aprendente a gerenciar sua própria aprendizagem, sem requerer a presença do professor para a busca do conhecimento. Assim, o professor ajudará o aluno a se tornar mais independente, mais consciente e mais responsável na busca e aquisição de conhecimento.

Na proposta por nós sugerida, o professor oferece ao aluno a opção de escolha para a realização da tarefa e sinaliza possibilidades para a busca de novos conhecimentos (expansão). Nesse sentido, estamos estimulando nossos alunos a repensarem seus papeis como aprendizes ativos na aprendizagem de uma língua estrangeira, na tentativa de monitorarem e avaliarem seu progresso e suas dificuldades dentro e fora de um contexto social de ensino.

\section{TAREFA 2: Lançar um outro olhar sobre filmes}

Em nossas práticas de ensino de inglês como língua estrangeira (EFL) temos percebido que o interesse dos aprendentes aumenta quando utilizamos partes de filmes e de programas televisivos, em qualquer fase do processo de aprendizagem. Vivemos em um 
mundo dominado pela imagem, mais particularmente pela imagem em movimento. Filmes atraem os alunos pela forma peculiar que têm de contar uma história (Stempleski \& Tomalin, 2001), além de serem excelentes recursos para estimular alunos a planejarem suas próprias atividades de aprendizagem. Pensando nisso, propusemos a utilização de duas worksheets nas quais os alunos são estimulados a avaliar, escolher e expandir seus conhecimentos por meio dos filmes.

Na primeira parte da tarefa, sugerimos que eles conversem com os colegas de classe sobre os seus filmes preferidos e preencham as informações na worksheet apresentada a seguir. Esse é um bom momento para incentivá-los a adquirir um olhar mais crítico sobre suas preferências cinematográficas.

\section{Worksheet 1}

\begin{tabular}{|l|l|l|}
\hline \multicolumn{3}{|c|}{ BEST FILM SURVEY } \\
\hline What's the best film you have ever seen? & Reasons \\
\hline Student's name & Film & \\
\hline 1. & & \\
\hline 2. & & \\
\hline 3. & & \\
\hline
\end{tabular}

( Adaptado de Stempleski \& Tomalin, 2001:14 )

Já na segunda parte da tarefa, os aprendentes terão a chance de aprofundar a avaliação dos filmes por meio da observação das questões a serem analisadas. Ao final, eles terão também a oportunidade não somente de buscar novos conhecimentos como de acrescentar informações ao MD utilizado - neste caso, os filmes - ao sugerirem modificações no final da história.

Sugerimos que primeiramente os alunos preencham a worksheet e que, somente depois, troquem as informações contidas nela com seus colegas em sala de aula. Desta forma, terão a oportunidade de compartilhar ideias sobre filmes diferentes, integrando habilidades de compreensão e produção oral e escrita em suas práticas. 


\section{Worksheet 2}

\begin{tabular}{|l|l|}
\hline \multicolumn{2}{|c|}{ TALK ABOUT THE STORY / SCENE } \\
\hline Film chosen: & \\
\hline $\begin{array}{l}\text { PLOT (What are the main things that happen in } \\
\text { the story?) }\end{array}$ & \\
\hline MAIN CHARACTERS (What are their names?) & \\
\hline SETTING (Where does the story take place?) & \\
\hline $\begin{array}{l}\text { POINT OF VIEW ( Who is the narrator of the } \\
\text { story?) }\end{array}$ & \\
\hline GENRE ( What is the genre of the film?) & \\
\hline THEME (What is the theme of the film?) & \\
\hline $\begin{array}{l}\text { YOUR CHOICE (If you were the director of the } \\
\text { film and could change its end, how would it be?) }\end{array}$ & \\
\hline
\end{tabular}

( Adaptado de Stempleski \& Tomalin, 2001:55 )

Acreditamos que a utilização de tais recursos para fins didáticos facilita a compreensão, permite a prática das quatro habilidades, oferece oportunidades para discutir aspectos culturais, além de ser uma forma criativa e inspiradora de promover a comunicação e a autonomia (Fernandes \& Menezes, 2008).

TAREFA 3: Planejar seu tempo e atividades de estudo

Quase todos os livros de ensino de LE apresentam uma tarefa na qual os alunos precisam descrever o seu dia. Essa atividade geralmente demanda o preenchimento de um quadro com os horários e o que cada aluno faz em cada momento do seu cotidiano. Ao analisar essa tarefa, conclui-se que ela não é instigadora para o aluno, sendo que às vezes, este não se sente à vontade para revelar seus hábitos diários diante dos colegas. Mesmo que o faça, é difícil identificar qual seria o significado dessa tarefa para sua vida e para sua aprendizagem, uma vez que ninguém enumera para o interlocutor todas as suas atividades em uma demonstração clara de que o que se aprende na aula não é transferível para a interação real. 
No lugar dessa tarefa, propomos uma modificação, a qual visa provocar a conscientização a respeito da ação de aprender, conforme podemos ver na atividade a seguir. Hipoteticamente, pensemos em um aprendente que frequenta aulas de inglês no turno da manhã, ao qual se pede que execute as seguintes ações:

\begin{tabular}{|l|l|}
\hline Time & Activity \\
\hline 1 p.m. & While on the bus, going home, recall what I learned in class. \\
\hline 4 p.m. & Do the homework. \\
\hline 6 p.m. & $\begin{array}{l}\text { Expand what I learned today by finding a connection to my own life. } \\
\text { Think about what I did not learn well and } \\
\text { what I should ask the teacher or colleagues who are more competent } \\
\text { than I am. } \\
\text { which resources I can use to solve my own learning problems. }\end{array}$ \\
\hline Next day & Do the things I thought about last evening. \\
\hline
\end{tabular}

Ao refletirem sobre o que fazem para aprender, os aprendentes inevitavelmente pensarão se o tempo e o esforço despendidos são suficientes e se as estratégias utilizadas são, de fato, as mais adequadas. O professor pode também propor que eles discutam em grupos, na aula seguinte, o modo como fizeram as atividades e o que acharam de seu desempenho. Assim, o professor estará fomentando práticas autônomas de seus alunos.

\section{CONSIDERAÇÕES FINAIS}

Como vimos anteriormente, nas três tarefas exemplificadoras, os materiais didáticos podem ser utilizados pelo professor para fomentar a autonomia dos aprendentes. Para tal, é preciso que as tarefas sejam escolhidas ou adaptadas de modo a engajá-los em uma prática social e comunicativa de ensino de LE, respeitando, assim, as individualidades e estilos de aprendizagem de cada um. Na Tarefa 1, deixamos à escolha do aluno qual site acessar e, em seguida, o próprio aluno expandiria sua pesquisa acessando outros sites de sua preferência, sem, no entanto, ficar desorientado em sua tarefa, uma vez que ele seria apoiado pelo checklist. Na Tarefa 2, houve também a prática da escolha e da expansão, especialmente pelos alunos terem tido a oportunidade de acrescentar algo diferente à tarefa, ao criarem um final diferente para o filme analisado. Na Tarefa 3, a atividade proposta pelo livro didático 
foi substituída por outra que tocasse mais os interesses do aluno, transformando-a em uma atividade reflexiva, autoregulatória e propiciadora de autonomia.

Dessa forma, podemos verificar que materiais didáticos fomentadores de autonomia fazem valer a voz do aprendente. Frequentemente eles promovem práticas de escolhas envolvendo tomada de consciência, posicionamento crítico e reflexão, as quais fornecem ao aprendente oportunidades para trabalhar de forma mais independente fora do contexto de sala de aula.

\section{REFERÊNCIAS}

Benson, P. (2001). Teaching and researching autonomy in language learning. Harlow: Logman.

Benson, P. \& Huang, J. (2008). Autonomy in the transition from foreign language learning to foreign language teaching. Revista DELTA, vol. 24 - $\mathrm{n}^{0}$ especial, São Paulo: LAEL/PUC-SP.

Breen, M.P. (1987). Learner contributions to task design. In: Candlin, C.N. \& Murphy, D.F. (eds.). Language learning tasks. UK: Prentice-Hall International Ltd and Lancaster University.

Dickinson, L. (1987). Self-instruction in language learning. Cambridge: Cambridge University Press.

. (1994). Learner autonomy: what, why and how? In: Leffa, V.J. Autonomy in language learning. Porto Alegre: Editora Universidade/UFRGS.

Fernandes, E.C.de S. \& Menezes, C. A. (2008). English Teaching Development: focus on the use of authentic video activities as a motivational resource to enhance EFL learning. Trabalho apresentado no II Congresso Latino-Americano de Formação de Professores de Línguas. Rio de Janeiro: PUC-RJ.

Holec, H. (1981). Autonomy in Foreign Language Learning. Oxford: Pergamon.

Magno e Silva, W. (2008). A model for the enhancement of autonomy. Revista DELTA, vol. 24 - $\mathrm{n}^{0}$ especial. São Paulo: LAEL/PUC-SP.

Paiva, V. M. de O. (2005). Autonomia e complexidade: uma análise de narrativas de aprendizagem. In: Freire, M. M; Abrahão, M.H.V. \& Barcelos, A.M.F. (orgs.). Linguística Aplicada e contemporaneidade. Campinas: Pontes.

Stempleski, S. \& Tomalin, B. (2001). Film. New York: Oxford University Press. 
ANEXO

CHECKLIST FOR EVALUATING WEBSITES

\begin{tabular}{|l|l|l|}
\hline \multicolumn{1}{|c|}{ AUTHORITY } & YES & NO \\
\hline Is the information reliable? & & \\
\hline Is the author an expert on the subject? & & \\
\hline Does the resource have a reputable organization or expert behind it? & & \\
\hline Are the sources of information stated? Can you verify the information? & & \\
\hline Can the author be contacted for clarification? & & \\
\hline $\begin{array}{l}\text { Does the author list his/her name? (if you don't know who the author is it's best } \\
\text { not to use the material) }\end{array}$ & & \\
\hline
\end{tabular}

\begin{tabular}{|l|c|c|}
\hline \multicolumn{1}{|c|}{ SCOPE } & YES & NO \\
\hline Is the material at this site useful, unique, accurate? & & \\
\hline Is it derivative, repetitious or doubtful? & & \\
\hline Is the purpose of the resource clearly stated? Does it fulfill its purpose? & & \\
\hline Are the items included in the resource? & & \\
\hline Are subject area, time period, or types of material covered? & & \\
\hline Is the information factual? & & \\
\hline Is the information opinion? & & \\
\hline Does the site contain original information or simply links? & & \\
\hline Is the resource updated? & & \\
\hline Does the site have clear and obvious pointers to new content? & & \\
\hline Are the links up-to-date? & \\
\hline
\end{tabular}

\begin{tabular}{|l|c|c|}
\hline \multicolumn{1}{|c|}{ FORMAT AND PRESENTATION } & YES & NO \\
\hline Is the information easy to get to? & & \\
\hline Do the links get to something useful? & & \\
\hline Do the graphical images enhance the resource? & & \\
\hline Do the graphical images distract from the content? & & \\
\hline Is the target audience or intended users clearly indicated? & & \\
\hline Is the arrangement of links uncluttered? & & \\
\hline Does the site have its own search engine? & & \\
\hline Is the site easily browsable or searchable? & \\
\hline
\end{tabular}

\begin{tabular}{|l|c|c|}
\hline \multicolumn{1}{|c|}{ ACCESSIBILITY } & YES & NO \\
\hline Are the web pages' hyperlinks taking you to “not found” pages? & & \\
\hline Do the connected hyperlinks bring you to advertisements? & & \\
\hline Does the 'official' page of a subject link to lots of irrelevant web site? & & \\
\hline
\end{tabular}

(Adaptado de http://library.usm.maine.edu/research/researchguides/webeval.php?ID=0.

Acesso em 10/05/2009) 


\section{OS AUTORES}

Walkyria Magno e Silva é doutora em Ciências da Linguagem pela Universidade de Toulouse le Mirail, França e Mestre em Linguística pela UNICAMP. Ensina no Curso de Letras, Licenciatura em Inglês e no Programa de Mestrado em Letras da UFPA. Coordena o projeto de pesquisa "Caminhos da Autonomia na Aprendizagem de Línguas Estrangeiras: o papel da motivação". Ela insiste em dizer que continua aprendendo.

e-mail: wmagno@ufpa.br

Edwiges Conceição de Souza Fernandes é professora de língua inglesa e coordenadora dos Cursos Livres de Idiomas - UEPA. Possui mestrado em Linguística, especialização em Linguística Aplicada, graduação em Letras - inglês e português e certificado de proficiência em inglês pela Universidade de Michigan. Atua principalmente nas seguintes áreas: língua inglesa; ESP; literaturas inglesa e americana.

e-mail: edwigesfernandes@hotmail.com

Marcus de Souza Araújo é Mestre em Letras (Linguística) pela UFPA e especialista em inglês pela PUC-MG. É professor de inglês da Universidade Federal do Pará. Suas áreas de interesse incluem ESP, leitura em LE, livro didático, gêneros textuais e preparação de material didático.

e-mail: marcusaraujo@interconect.com.br 